\title{
Using lithium to estimate ages for solar-type stars
}

\author{
David R. Soderblom ${ }^{1}$ \\ ${ }^{1}$ Space Telescope Science Institute, \\ 3700 San Martin Drive, Baltimore MD 21218 USA \\ email: drs@stsci.edu
}

\begin{abstract}
Can observations of $\mathrm{Li}$ in F, G, and $\mathrm{K}$ stars be used to derive an age for an individual star or a group? This is a brief progress report on using Li for age estimation in PMS and ZAMS stars.
\end{abstract}

Keywords. Stars:ages, solar-type, convection

The decline of the surface $\mathrm{Li}$ abundance with age is well known for stars near and below $1 M_{\odot}$. Observations and models of this phenomenon should provide constraints on convection in these stars, the process believed to be the underlying cause of the $\mathrm{Li}$ depletion. This is a progress report on an effort to invert the problem to use observations of $\mathrm{Li}$ in $\mathrm{F}, \mathrm{G}$, and $\mathrm{K}$ dwarfs to estimate their ages.

A full understanding of Li depletion involves some complex and not well understood physics and requires accurate determinations of basic stellar quantities such as temperature and composition. Also, transforming an observation of $\mathrm{Li}$ to an abundance is very sensitive to temperature as well as non-LTE effects. However, when estimating ages these quantities are often only poorly constrained and I wished to address a simpler question: If observations of the Li equivalent width $\left(W_{\lambda}\right)$ and color $((B-V)$, say, or the equivalent derived from another color or from $\left.T_{\text {eff }}\right)$ are at hand, how well can an age be estimated? Can an age be determined at all for a single star, or is some minimum number in a group required?

The data used will be described fully and illustrated in a forthcoming paper, and here I will summarize the key points:

- The youngest stars with good Li observations are T Tauri stars in Taurus-Auriga, the Orion Nebula Cluster, and NGC 2264, all with ages of 5 Myr or less. The scatter in $W_{\lambda}$ among these stars is small and consistent with the observational errors. The consistency in $\mathrm{Li}$ among these different groups suggests stars in the present epoch all form with the same initial $\mathrm{Li}$ abundance, and the agreement with the primordial solar Li abundance (from meteorites) indicates that that value has not changed significantly in $\sim 5$ Gyr.

- By contrast, the Pleiades is a well-observed cluster with an age of $125 \mathrm{Myr}$ and it exhibits an inherent spread in $W_{\lambda}$ that grows in going to cooler stars, becoming as much as 1 dex for $\mathrm{K}$ dwarfs (see Fig. 1). This spread is much larger than the observing errors and also significantly larger than the scatter seen among $\mathrm{T}$ Tauri stars. The fact that $\mathrm{T}$ Tauris show little scatter implies that inhomogeneous stellar atmospheres (i.e., spots) are not the cause of the spread in $\mathrm{Li}$ in the Pleiades because the $\mathrm{T}$ Tauris are expected to be at least as spotted as ZAMS stars.

- The run of $W_{\lambda}$ with color for the $\alpha$ Persei cluster and NGC 2451 at about 80 Myr is indistinguishable from that of the Pleiades (see Fig. 2). This implies that Li depletion at least on average - goes through plateau stages where little or nothing happens for an extended time. 


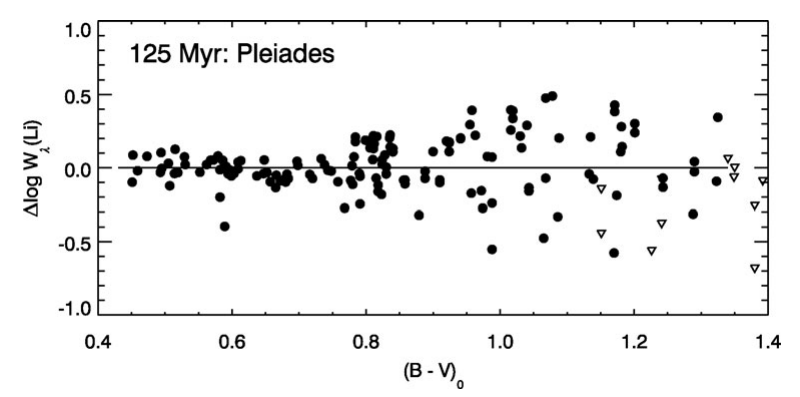

Figure 1. Lithium equivalent widths for F, G, and $\mathrm{K}$ stars in the Pleiades. A mean relation has been fitted and subtracted to show how the scatter in $W_{\lambda}$ varies with color.

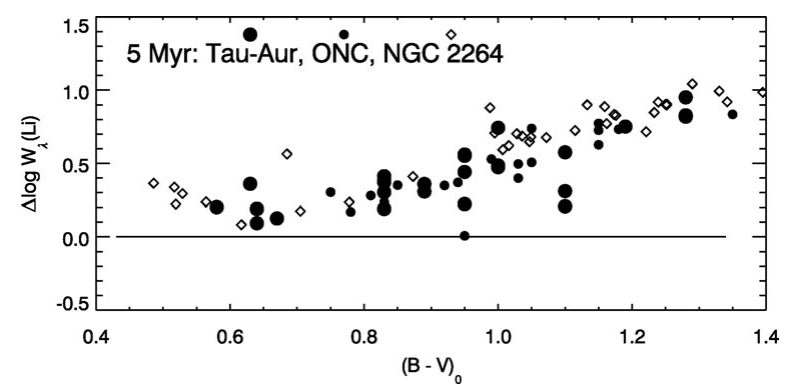

Figure 2. Li equivalent widths for $\mathrm{T}$ Tauri stars in Tau-Aur, the Orion Nebula Cluster, and NGC 2264, relative to the Pleiades.

- There are clusters with ages between those of the T Tauris and $\alpha$ Persei but they are sparse and so the data are less well defined. Nevertheless, $W_{\lambda}$ declines in progression with age as expected, although there can again be plateaus where little difference in the average $W_{\lambda}$ is seen despite a significant difference in age.

- Li continues to decline in stars after they reach the ZAMS, as seen in 200 Myr-old clusters (M35, NGC 2516 and Blanco 1), and at 300 Myr (M34 and NGC 6475).

- Stars in the Hyades and other clusters of that age (Praesepe, Coma, and NGC 6633) have well-defined runs of $W_{\lambda}$ with color and with little scatter. This suggests that the large scatter seen in the Pleiades may later lessen, but there is no known mechanism to achieve that. Also, stars in the 4 Gyr-old cluster M67 show an appreciable spread in Li. The Hyades may possibly be misleading because it is metal-rich.

There are trends of declining average $W_{\lambda}$ for the $\mathrm{F}$ and $\mathrm{G}$ stars but they are comparable to the scatter seen. For the $\mathrm{K}$ dwarfs, however, the decline in $W_{\lambda}$ is large and rapid up to 200-300 Myr or so. The large scatter seen among stars of a single age (notably the Pleiades) means that for a single star one can only set limits to age, but if data for a group of 10-20 stars exist then a more precise age can be set. This makes it possible to use observations of $\mathrm{Li}$ in $\mathrm{K}$ dwarfs to at least set the age ordering of young kinematic groups such as $\beta$ Pictoris, $\eta$ Cha, TW Hya, etc. 